\title{
Max Cut and the Smallest Eigenvalue
}

\author{
LuCA TREvisAN*
}

January 10, 2019

\begin{abstract}
We describe a new approximation algorithm for Max Cut. Our algorithm runs in $\tilde{O}\left(n^{2}\right)$ time, where $n$ is the number of vertices, and achieves an approximation ratio of .50769 . On instances in which an optimal solution cuts a $1-\varepsilon$ fraction of edges, our algorithm finds a solution that cuts a $1-4 \varepsilon^{1 / 3}-o(1)$ fraction of edges.

Our main result is a variant of spectral partitioning, which can be implemented in nearly linear time, that, given a graph in which the Max Cut optimum is a $1-\varepsilon$ fraction of edges, finds a set $S$ of vertices and a bipartition $L, R=S-L$ of $S$ such that at least a $1-4 \sqrt{\varepsilon}$ fraction of the edges incident on $S$ have one endpoint in $L$ and one endpoint in $R$. (This can be seen as an analog of Cheeger's inequality for the smallest eigenvalue of the adjacency matrix of a graph.) Iterating this procedure yields the approximation results stated above.

The algorithm of Goemans and Williamson [GW95], together with the SDP solver of Arora and Kale [AK07], give an approximation ratio of .878 in nearly linear time. While our algorithm is inferior in both running time and approximation ratio, it is the first algorithm to achieve an approximation better than $1 / 2$ for Max Cut by any means other than using a hyperplane to round the solution to a semidefinite program.
\end{abstract}

\section{Introduction}

In the Max CUT problem, we are given an undirected graph with non-negative weights on the edges and we wish to find a partition of the vertices (a cut) which maximizes the weight of edges whose endpoints are on different sides of the partition (such edges are said to be cut by the partition). We refer to the cost of a solution as the fraction of weighted edges of the graph that are cut by the solution.

It is easy, given any graph, to find a solution that cuts half of the edges, providing an approximation factor of $1 / 2$ for the problem. The algorithm of Goemans and Williamson [GW95], based on a Semidefinite Programming (SDP) relaxation, has a performance ratio of $.878 \cdots$ on general graphs, and it finds a cut of cost $1-O(\sqrt{\varepsilon})$ in graphs in which the optimum is $1-\varepsilon$. Assuming the unique games conjecture, both results are best possible for polynomial time algorithms [Kho02, KKMO04, MOO05] (see also [OW08]). Arora and Kale [AK07] show that the Goemans-Williamson SDP relaxation can be near-optimally solved in nearly linear time in graphs of bounded degree (or more

\footnotetext{
*Iuca@cs.berkeley.edu. U.C. Berkeley, Computer Science Division. This material is based upon work supported by the National Science Foundation under grant No. CCF-0729137 and by the BSF under grant 2002246.
} 
generally, in weighted graphs with bounded ratio between largest and smallest degree). We show in Appendix A.1 that, using a reduction [Tre01], the Arora-Kale algorithm can be used to achieve the approximation performance of the Goemans-Williamson algorithm on all graphs in nearly-linear time.

No method other than SDP is known to yield an approximation better than $1 / 2$, and such approximation has been rules out for large classes of Linear Programming Relaxations [dlVKM07, STT07].

\section{Our Results}

Our main result is a variant of the spectral partitioning algorithm with the following property: given a graph $G$ in which the Max CUT optimum cost is $1-\varepsilon$, it finds two disjoint sets of vertices $L, R$ such that the number of edges with one endpoint in $L$ and one endpoint in $R$ is at least a $1-4 \sqrt{\varepsilon}$ fraction of the total weight of edges incident ${ }^{1}$ on $L \cup R$. (See Theorem 1.)

To derive an approximation algorithm for Max CUT, given a graph we apply the partitioning algorithm and find sets $L, R$ as above. If the number of edges with one endpoint in $L$ and one in $R$ is smaller than half the total number of edges incident on $L \cup R$, then we randomly partition the graph. Otherwise, we remove the vertices in $L \cup R$ from the graph, recursively find a partition of the residual graph, and then put back the vertices of $L$ on one side of the partition and vertices of $R$ on the other side.

In the analysis, we need to bound the number of edges which are cut by our algorithm: at the end of the recursion, only half of the edges of the residual graph are cut, but the stopping condition implies that the optimal of the residual graph is small (and, if the optimum of the original graph was very close to 1 , that the residual graph itself must be small), so the number of edges in the final residual graph cut by our algorithm is not much smaller than the corresponding number cut by an optimal solution. Regarding the rest of the execution of the algorithm, we mantain the invariant that a large fraction of the edges that we remove from the graph will be cut once we put back the sets $L$ and $R$ into the solution. In general graphs, this leads to a .507 approximation. In graphs in which the optimum Max CUT has cost $\geq 1-\varepsilon$, our algorithm finds a cut of cost $\geq 1-4 \varepsilon^{1 / 3}$.

\section{Relation to Cheeger's Inequality}

In the case of regular graphs, our main result, Theorem 1, may be seen as an analog of Cheeger's inequality [Alo86] for the smallest (rather than second largest) eigenvalue of the adjacency matrix of the graph. We discuss this analogy in Section 5

\section{Relation to the Goemans-Williamson Relaxation}

Our algorithm may also be seen as a primal-dual algorithm that produces, along with a cut, a feasible solution to the semidefinite dual of the Goemans-Williamson relaxation such that the cost of the cut is at least .507 times the cost of the dual solution. We describe this view in Section 6 .

We know of only one other primal-dual approximation algorithm based on Semidefinite Programming: one of the algorithms for uniform sparsest cut of Arora, Rao, and Vazirani [ARV04].

\footnotetext{
${ }^{1}$ We say than an edges $(i, j)$ is incident on a set of vertices $S$, if at least one of $i$ or $j$ belongs to $S$.
} 


\section{Other Relations to Previous Work}

It has been known that one can use spectral methods to certify an upper bound to the Max CUT optimum of a given graph. In particular, in $d$-regular graph whose adjacency matrix has eigenvalues $d=\lambda_{1} \geq \lambda_{2} \geq \cdots \geq \lambda_{n}$, one can use the Expander Mixing Lemma to see that the Max CUT optimum is at most

$$
\frac{1}{2}+\frac{1}{2} \cdot \max \left\{\frac{\lambda_{2}}{d}, \frac{\left|\lambda_{n}\right|}{d}\right\}
$$

A more careful analysis shows that one needs not refer to $\lambda_{2}$, and that, indeed, the Max CUT optimum is at most

$$
\frac{1}{2}+\frac{1}{2} \cdot \frac{\left|\lambda_{n}\right|}{d}
$$

as was noted, for example, by Feige and Ofek [FO05], and possibly before. (This is essentially our Lemma 2.)

What is new is that we are able to prove a converse, in Lemma 3, and show that a non-trivial consequence follows whenever $\left|\lambda_{n}\right|$ is close to $d$.

As mentioned above, it was known that if $G$ is $d$-regular, then $\lambda_{n}=-d$ if and only if $G$ has a bipartite connected component. In particular, if $G$ is connected and not bipartite then $\lambda_{n}>-d$. Alon and Sudakov [AS00] consider the question of how small, in such case, can the gap $d-\left|\lambda_{n}\right|$ be. They show that, if $G$ is connected and not bipartite, it has maximum degree $d$ and diameter $D$, then $d-\left|\lambda_{n}\right| \geq \frac{1}{(D+1) \cdot n}$. Our result implies the weaker bound $d-\left|\lambda_{n}\right| \geq \frac{1}{d n^{2}}$ in a $d$-regular graph. The idea of iteratively removing parts of an instance in which one has a good solution appears in various works on the sparsest cut problem (for example in the way Spielman and Teng [ST04] find a balanced separator using their "nibble" procedure), and it was used to approximate the Max CUT problem (in the version in which one wants to minimize the number of uncut vertices) by Agarwal et al. [ACMM05]. In the algorithm of Agarwal et al., as in our algorithm, the basic procedure that is being iterated finds a set $S$ of vertices and a bipartition $L, R$ of $S$ such that most of the edges incident on $S$ have one endpoint in $L$ and one endpoint in $R$.

\section{Sparsification}

It follows from the Chernoff Bound that if we are given a graph $G=(V, E)$ and we sample $O\left(\delta^{-2}|V|\right)$ edges with replacement ${ }^{2}$ then, with high probability, every cut $(S, \bar{S})$ has the same cost in the original graph as in the new graph, up to an additive error $\delta .^{3}$

For this reason, all the dependency on $|E|$ in the running time of our algorithm can be changed to a dependency on $|V|$ with an arbitrarily small loss in the approximation factor.

\footnotetext{
${ }^{2}$ If the graph is unweighted, we sample from the uniform distribution over the edges; otherwise we sample from the distribution in which each edge has a probability proportional to its weight.

${ }^{3}$ Note that the sparsified graph is an unweighted multigraph, and that the sparsification process is considerably simpler than the one used for algorithms for sparsest cut and other graph minimization problems.
} 


\section{The Spectral Algorithm}

In this section we prove our main result.

Theorem 1 (Main) There is an algorithm that, given a graph $G=(V, E)$ for which the optimum of the Max CUT problem is at least $1-\varepsilon$, and a parameter $\delta$, finds disjoint sets $L, R \subseteq V$ such that at least a $1-4 \sqrt{\varepsilon}-\delta$ fraction of the edges incident on $L \cup R$ have one endpoint in $L$ and one endpoint in $R$.

The algorithm can be implemented in nearly-linear randomized time $O\left(\delta^{-2} \cdot(|V|+|E|) \cdot \log |V|\right)$.

Let $A$ be the adjacency matrix of our input graph $G$ (hence $A_{i, j}$ is the weight of the edge between $i$ and $j$ ), and $D$ be the diagonal matrix such that $D_{i, i}$ is the degree $d_{i}$ of vertex $i$ and $D_{i, j}=0$ for $i \neq j$.

Theorem 1 follows by combining the following two results, and noting that, for $a, b \geq 0, \sqrt{a+b} \leq$ $\sqrt{a}+\sqrt{b}$.

Lemma 2 If the optimum Max CUT in $G$ has cost at least $1-\varepsilon$, there is a vector $x \in \mathbb{R}^{V}$ such that

$$
x^{T}(D+A) x \leq 2 \varepsilon \cdot x^{T} D x .
$$

Furthermore, for every $\delta>0$, we can find in time $O\left(\delta^{-1} \cdot(|E|+|V|) \cdot \log |V|\right)$ a vector $x \in \mathbb{R}^{V}$ such that

$$
x^{T}(D+A) x \leq(2 \varepsilon+\delta) \cdot x^{T} D x
$$

Lemma 3 Given a vector $x \in \mathbb{R}^{V}$ such that $x^{T}(D+A) x \leq \varepsilon \cdot x^{T} D x$, we can find in time $O(|E|+$ $|V| \log |V|)$ two disjoint sets $L, R \subseteq V$ such that at least a $1-\sqrt{8 \varepsilon}$ weighted fraction of the edges incident on $L \cup R$ has one endpoint in $L$ and one endpoint in $R$.

Lemma 2 has a simple proof, and it can be seen as a statement about the semidefinite dual of the Goemans-Williamson relaxation, as discussed in Section 6. Lemma 3 is the main result of this paper.

\subsection{Proof of Lemma 2}

Consider the optimization problem

$$
\min _{x \in \mathbb{R}^{V}} \frac{x^{T} A x}{x^{T} D x}
$$

Let $(S, \bar{S})$ be an optimum cut for $G$, and define the vector $x^{*} \in\{-1,1\}^{V}$ such that $x_{i}^{*}=1$ if $i \in S$ and $x_{i}^{*}=-1$ otherwise. Then $x^{* T} A x^{*}$ equals twice the difference between the number of edges not cut by $(S, \bar{S})$ and the number of edges that are cut, which is at most $2 \cdot(2 \varepsilon-1) \cdot|E|$. As for $x^{* T} D x^{*}$, we have

$$
x^{* T} D x^{*}=\sum_{i} d_{i} \cdot\left(x_{i}^{*}\right)^{2}=\sum_{i} d_{i}=2 \cdot|E|
$$


Thus $x^{*}$ is a feasible solution to (1) of cost at most $2 \varepsilon-1$, and if $\hat{x}$ is the optimal solution to (1), then we must have

$$
\hat{x}^{T} A \hat{x} \leq(2 \varepsilon-1) \hat{x}^{T} D \hat{x}
$$

To prove the "furthermore" part of the lemma, we observe that the optimization problem in (1) is equivalent to

$$
\min _{x \in \mathbb{R}^{V}} \frac{x^{T} D^{-1 / 2} A D^{-1 / 2} x}{x^{T} x}
$$

where $D^{-1 / 2}$ is the matrix that such that $D_{i, j}^{-1 / 2}=0$ if $D_{i, j}=0$, and $D_{i, j}^{-1 / 2}=1 / \sqrt{D_{i, j}}$ otherwise. In turn, the optimization problem in (2) is the problem of computing the smallest eigenvalue of $D^{-1 / 2} A D^{-1 / 2}$, which is the same as computing the largest eigenvalue of the positive semidefinite matrix $I-D^{-1 / 2} A D^{-1 / 2}$.

Given a $n \times n$ positive semidefinite matrix $M$ with $T$ non-zero entries and of largest eigenvalue $\lambda_{1}$, and a parameter $\delta$, it is possible to find a vector $x$ such that $x^{T} M x \geq \lambda_{1} \cdot(1-\delta) \cdot x^{T} x$ in randomized time $O\left(\delta^{-1} \cdot(T+n) \cdot \log n\right)$ [KW92]. Applying the algorithm to $I-D^{-1 / 2} A D^{-1 / 2}$, which, as proved above, has a largest eigenvalue which is at least $2-2 \varepsilon$, and which has $|E|+|V|$ non-zero entries, we find in randomized time $O\left(\delta^{-1 / 2} \cdot(|E|+|V|) \cdot \log |V|\right)$ a vector $x^{\prime}$ such that

$$
\frac{x^{T}\left(I-D^{-1 / 2} A D^{-1 / 2}\right) x^{\prime}}{x^{\prime T} x^{\prime}} \geq 2-2 \varepsilon-\delta
$$

and so

$$
x^{\prime T} D^{-1 / 2} A D^{-1 / 2} x^{\prime} \leq(2 \varepsilon+\delta-1) \cdot x^{\prime T} x^{\prime}
$$

and, if we define $x^{\prime \prime}:=x^{\prime} D^{1 / 2}$, then

$$
x^{\prime \prime T} A x^{\prime \prime} \leq(2 \varepsilon+\delta-1) x^{\prime \prime T} D x^{\prime \prime}
$$

which we can rewrite

$$
x^{\prime \prime T}(A+D) x^{\prime \prime} \leq(2 \varepsilon+\delta) x^{\prime \prime T} D x^{\prime \prime}
$$

\subsection{Proof of Lemma 3}

We now come to our main result.

The condition $x(D+A) x \leq \varepsilon \cdot x D x$ is equivalent to

$$
\frac{1}{2} \sum_{i, j} A_{i, j}\left(x_{i}+x_{j}\right)^{2} \leq \varepsilon \sum_{i} d_{i} x_{i}^{2}
$$

Before starting the formal proof, we describe a heuristic argument that gives some intuition for the actual proof. 
Proof Idea. Equation (3) states that the average value of $\left(x_{i}+x_{j}\right)^{2}$, for an edge $(i, j)$, is at most $\varepsilon$ times the average value of $x_{i}^{2}$ and $x_{j}^{2}$. So, non-rigorously, we would guess that for a typical edges the value of $\left|x_{i}+x_{j}\right|$ is at most about $\sqrt{\varepsilon}$ times $\left|x_{i}\right|+\left|x_{j}\right|$. For this to happen, it must be the case that $x_{i}$ and $x_{j}$ have different signs, and their absolute value is nearly the same; that is, for some positive $c, x_{i}=-c$ and $x_{j}=c(1-\sqrt{\varepsilon})$. Suppose now that we pick a random threshold $t$, and we define $L:=\left\{i: x_{i} \leq-t\right\}$ and $R:=\left\{j: x_{j} \geq t\right\}$. Then the probability that the edge $(i, j)$ is incident on $L \cup R$ is proportional to $c$, while the probability that the edge is incident on $L \cup R$ but fails to have one endpoint in $L$ and one endpoint in $R$ is proportional to $c \sqrt{\varepsilon}$ (it can only happen when $t$ is between $c(1-\sqrt{\varepsilon}$ ) and $c$, in which case $j \in R$ but $i \notin L)$. So we expect only about a $\sqrt{\varepsilon}$ fraction of the edges incident on $L \cup R$ to fail to have one endpoint in $L$ and one endpoint in $R$.

Our algorithm, which we call the 2-Thresholds Spectral Cut algorithm and abbreviate 2TSC, is as follows:

- Algorithm 2TSC

- For every vertex $i$

- Define $L_{i}:=\left\{j: x_{j} \leq-\left|x_{i}\right|\right\}, R_{i}:=\left\{j: x_{j} \geq\left|x_{i}\right|\right\}$

- Let $M_{i}$ be the weighted number of edges incident on $L_{i} \cup R_{i}$, and $C_{i}$ be the weighted number of such edges with one endpoint in $L_{i}$ and one endpoint in $R_{i}$

- Output the pair $\left(L_{i}, R_{i}\right)$ for which the ratio $C_{i} / M_{i}$ is largest

(The names $L$ and $R$ for the set are evocative of the fact that if we think of $x$ as an embedding of $V$ on the line, then $L$ is a set of leftmost vertices in the embedding, and $R$ is a set of rightmost vertices.)

The algorithm can be implemented to run in $O(|E|+|V| \log |V|)$ time. We first sort the vertices according to the value of $\left|x_{i}\right|$, and so we assume we have $\left|x_{1}\right| \leq\left|x_{2}\right| \leq \cdots \leq\left|x_{n}\right|$ when we run 2TSC. At each step, we maintain a count of how many edges have both endpoints in $L_{i}$, how many edges have both endpoints in $R_{i}$, how many edges cross the partition, how many edges connect $L_{i} \cup R_{i}$ with the rest of the graph, and how many edges are not incident on $L_{i} \cup R_{i}$. When we go from step $i$ to step $i+1$, we need to update all such quantities, and only the edges incident on $i$ or on $i+1$ move from one category to other. Step $i$, thus, take time $O\left(d_{i}+d_{i+1}\right)$, and all the $n$ steps together take time $O(|E|)$.

We need to argue that, under the assumption of the Lemma, the algorithm outputs a pair of sets $L_{i}, R_{i}$ such that $C_{i} \geq(1-\sqrt{8 \varepsilon}) \cdot M_{i}$.

In order to analyze $2 \mathrm{TSC}$, we study the following randomized process:

- Pick a value $t$ uniformly in $\left[0, \max _{i} x_{i}^{2}\right]$;

- Define $L:=\left\{j: x_{j} \leq-\sqrt{t}\right\}, R:=\left\{j: x_{j} \geq \sqrt{t}\right\}$

- Let $M$ be the weighted number of edges incident on $L \cup R$, and $C$ be the weighted number of edges with one endpoint in $L$ and one endpoint in $R$ 
Every pair $L, R$ generated by the probabilistic process is considered by algorithm 2TSC at some stage; this implies that if algorithm 2TSC produces a pair $L_{i}, R_{i}$ such that $C_{i}<(1-\sqrt{8 \varepsilon}) M_{i}$, then in the randomized process we must have $C<(1-\sqrt{8 \varepsilon}) M$ with probability 1 and, in particular, $\mathbb{E} C<(1-\sqrt{8 \varepsilon}) \mathbb{E} M$. We shall prove that $\mathbb{E} C \geq(1-\sqrt{8 \varepsilon}) \mathbb{E} M$ and so we shall conclude that the output of algorithm 2TSC satisfies the Claim.

Since Equation (3) is scale invariant, we may assume that $\max _{i}\left|x_{i}\right|=1$, so that $t$ is chosen uniformly in $[0,1]$.

This means that, for a vertex $i$ with $x_{i}<0$, we have that $i$ can never belong to $R$, and it belongs to $L$ with probability $x_{i}^{2}$. A vertex $j$ with $x_{j}>0$ can never belong to $L$, and it belongs to $R$ with probability $x_{j}^{2}$. Define the random variable $W$ as the volume of the set $L \cup R$, that is, the sum of the degrees of the vertices in $L \cup R$. We immediately see that

$$
\mathbb{E} W=\sum_{i} d_{i} \cdot \mathbb{P}[i \in L \cup R]=\sum_{i} d_{i} x_{i}^{2}
$$

Since the number of edges incident on a set is at least half of the volume, we have

$$
\mathbb{E} M \geq \frac{1}{2} \sum_{i} d_{i} x_{i}^{2}
$$

It remains to estimate $C$. It is easier to work with $F:=M-C$, that is the weighted number of edges that are incident on $L \cup R$ but fail to have exactly one endpoint in $A$ and one endpoint in $B$. (Such edges may connect $L \cup R$ with the rest of the graph, or be completely contained in $L$, or be completely contained in $R$.) We call such edges failed edges.

A case analysis shows that, for every edge $(i, j)$,

$$
\mathbb{P}[(i, j) \text { fails }] \leq\left|x_{i}+x_{j}\right| \cdot\left(\left|x_{i}\right|+\left|x_{j}\right|\right)
$$

To verify Equation (4) we need to distinguish the case in which $x_{i}$ and $x_{j}$ have different signs from the case in which they have the same sign:

- If they have different signs, and, say, $\left|x_{i}\right|>\left|x_{j}\right|$, then the edge fails only when $\left|x_{j}\right|^{2} \leq t \leq\left|x_{i}\right|^{2}$. If $t<\left|x_{j}\right|^{2}$, then $i \in L$ and $j \in R$ (or vice versa), and the edge is cut, while if $t>\left|x_{i}\right|^{2}$ then $(i, j)$ is not incident on $L \cup R$. The probability that $(i, j)$ fails is then $\left|x_{i}\right|^{2}-\left|x_{j}\right|^{2}$ which is equal to the right-hand side of Equation (4).

- If they have the same sign, then the edge can never be cut, and so $(i, j)$ will fail whenever it is incident on $L \cup R$, that is, whenever $t \leq \max \left\{\left|x_{i}\right|^{2},\left|x_{j}\right|^{2}\right\}$. The probability that $(i, j)$ fails is thus $\max \left\{\left|x_{i}\right|^{2},\left|x_{j}\right|^{2}\right\}$, while the right-hand side of Equation (4) is $\left(\left|x_{i}\right|+\left|x_{j}\right|\right)^{2}$, which is always at least as large.

To complete our argument it remains to apply Cauchy-Schwarz and standard manipulations. 


$$
\begin{aligned}
\mathbb{E} F & =\frac{1}{2} \sum_{i, j} A_{i, j} \mathbb{P}[(i, j) \text { fails }] \\
& \leq \frac{1}{2} \sum_{i, j} A_{i, j}\left|x_{i}+x_{j}\right| \cdot\left(\left|x_{i}\right|+\left|x_{j}\right|\right) \\
& \leq \frac{1}{2} \sqrt{\sum_{i, j} A_{i, j}\left|x_{i}+x_{j}\right|^{2}} \cdot \sqrt{\sum_{i, j} A_{i, j}\left(\left|x_{i}\right|+\left|x_{j}\right|\right)^{2}}
\end{aligned}
$$

By our assumption,

$$
\sum_{i, j} A_{i, j}\left|x_{i}+x_{j}\right|^{2} \leq 2 \varepsilon \sum_{i} d_{i} x_{i}^{2}
$$

and it is a standard calculation that

$$
\sum_{i, j} A_{i, j}\left(\left|x_{i}\right|+\left|x_{j}\right|\right)^{2} \leq 2 \sum_{i, j} A_{i, j}\left(\left|x_{i}\right|^{2}+\left|x_{j}\right|^{2}\right)=4 \sum_{i} d_{i} x_{i}^{2}
$$

and so

$$
\mathbb{E} F \leq \frac{1}{2} \sqrt{8 \varepsilon} \sum_{i} d_{i} x_{i}^{2} \leq \sqrt{8 \varepsilon} \mathbb{E} M
$$

and

$$
\mathbb{E} C=\mathbb{E} M-F \geq(1-\sqrt{8 \varepsilon}) \mathbb{E} M
$$

This completes the proof that Algorithm 2TSC performs as required by the Lemma.

\section{Approximation for Max Cut}

In this section we analyze the following algorithm

- Algorithm: Recursive-Spectral-Cut

- Input: graph $G=(V, E)$, accuracy parameter $\delta$

- Run the algorithm of Theorem 1 with accuracy parameter $\delta$, and let $L, R$ be the sets found by the algorithm, $M$ the weighted number of edges incident on $L \cup R, C$ the weighted number of edges cut by $L, R$.

- If $|C| \leq \frac{1}{2}|M|$, then find a partition of $V$ that cuts $\geq|E| / 2$ edges, and return it.

- If $|C|>\frac{1}{2}|M|$, then let $G^{\prime}=\left(V^{\prime}, E^{\prime}\right)$ be the graph induced by $V^{\prime}:=V-(L \cup R)$, recursively call ReCURsive-Spectral-Cut on $G^{\prime}$, and let $V_{1}, V_{2}$ be the partion found by the algorithm; return $\left(V_{1} \cup L, V_{2} \cup R\right)$. 
Note that the algorithm runs in randomized time $O\left(\delta^{-2} \cdot|V| \cdot(|V|+|E|) \cdot \log |V|\right)$ because each iteration takes time $O\left(\delta^{-1} \cdot(|V|+|E|) \cdot \log |V|\right)$ and there are at most $|V|$ iterations.

First, we show that in general graphs algorithm RECURSIVE-SPECTRAL-CUT achieves an approximation ratio better than $1 / 2$. Let $\varepsilon_{0}=.015148 \cdots$ be the solution to $\frac{1}{2\left(1-\varepsilon_{0}\right)}=1-4 \sqrt{\varepsilon_{0}}$.

Theorem 4 Algorithm RECURSIVE-SPECTRAL-CUT achieves an approximation ratio which is at least $1 / 2\left(1-\varepsilon_{0}\right)-\delta=1-4 \sqrt{\varepsilon_{0}}-\delta=.507 \cdots-\delta$.

PRoOF: We proceed by induction on the number of recursive calls performed by the algorithm. Let opt be the cost of an optimum solution.

For the base case, suppose that there is no recursive call. Then, if we write opt $=1-\varepsilon$, the number of failed edges in the solution $L, R$ found in the first step must be more than half of the total number of edges incident on $L \cup R$, but our analysis shows that the number of failed edges can be at most a $4 \sqrt{\varepsilon}+\delta$ fraction, meaning that $4 \sqrt{\varepsilon}+\delta \leq 1 / 2$, and so

$$
\varepsilon \geq\left(\frac{1}{8}-\frac{\delta}{4}\right)^{2}>\frac{1}{64}-\frac{\delta}{16}
$$

this means that opt $\leq 63 / 64+\delta / 16$ and that the approximation ratio (recall that we return a cut of cost $1 / 2)$ is $1 / 2$ opt which is at least $32 /(64+4 \delta)$, which is more than $32 / 63-\delta$, and more than $.507 \cdots-\delta$.

For the inductive step, we consider two cases.

If the optimum is smaller than $1-\varepsilon_{0}$, then, considering that our algorithm always cuts at least half of all edges, we have an approximation ratio which is at least $1 / 2\left(1-\varepsilon_{0}\right)$ as required.

If the optimum is larger than or equal to $1-\varepsilon_{0}$, then the algorithm cuts at least a $1-4 \sqrt{\varepsilon_{0}}-\delta$ fraction of all edges incident on $L \cup R$, and, by inductive hypothesis, at least a $1-4 \sqrt{\varepsilon_{0}}-\delta$ of all edges than can possibly be cut from the residual graph $G^{\prime}$, and so it cuts at least a $1-4 \sqrt{\varepsilon_{0}}-\delta$ fraction of the edges cut by an optimal solution to $G$.

The analysis can be improved when we apply the algorithm on graphs on which the optimum is very large.

Theorem 5 If Algorithm RECURSIVE-SpeCtral-Cut receives in input a graph $G=(V$, E) whose optimum is $1-\varepsilon$, then it finds a solution that cuts at least a $1-4 \cdot \varepsilon^{1 / 3}-\delta$ fraction of edges.

Proof: We divide the execution of the algorithm into two phases: Phase 1 is the sequence of steps in which the number of edges of the residual graph is at least $4 \cdot \varepsilon^{1 / 3} \cdot|E|$, and Phase 2 is the sequence of remaining steps.

The edges which the algorithm fails to cut during Phase 2 is clearly at most $2 \cdot \varepsilon^{1 / 3} \cdot|E|$.

During Phase 1 , at each step we consider a residual graph $\hat{G}=(\hat{V}, \hat{E})$ that has $|\hat{E}| \geq 4 \varepsilon^{1 / 3}|E|$ edges, and we know that there is a feasible solution that cuts at least $|\hat{E}|-\varepsilon|E| \geq\left(1-\varepsilon^{2 / 3} / 4\right) \cdot|\hat{E}|$ edges. Thus, the sets $L, R$ found by algorithm 2 TSC are such that at most a $2 \varepsilon^{1 / 3}+\delta$ fraction of the edges incident on $L \cup R$ fail. Overall, the total number of failed edges during Phase 1 can be at most $\left(2 \varepsilon^{1 / 3}+\delta\right) \cdot|E|$. 


\section{Relation to Cheeger's Inequality}

In this section we compare our main result, Theorem 1, with Cheeger's inequality [Alo86]. We restrict our discussion to the case of regular graph.

If $G$ is a $d$-regular graph, and $A$ is its adjacency matrix, then $A$ has $n$ eigenvalues, counting multiplicities, which we shall call $\lambda_{1} \geq \lambda_{2} \geq \cdots \geq \lambda_{n}$. It is always the case that $\lambda_{1}=d$, and that $\left|\lambda_{i}\right| \leq d$ for every $i$. The extremal cases are captured by the following well-known facts:

1. $\lambda_{2}=d$ if and only if $G$ is disconnected, that is, if and only if there is a set $S,|S| \leq|V| / 2$, such that no edge of $G$ leaves $S$.

2. $\lambda_{n}=-d$ if and only if $G$ contains a bipartite connected component, that is, if and only if there is a set $S$ and partition of $S$ into disjoint sets $L, R$, such that all edges incident on $S$ have one endpoint in $L$ and one endpoint in $R$.

Cheeger's inequality characterizes the cases in which $\lambda_{2}$ is close to $d$. If there is a set $S,|S| \leq|V| / 2$ such that $\leq \varepsilon d|S|$ edges go between $S$ and $\bar{S}$, then $\lambda_{2} \leq d \cdot(1-2 \varepsilon)$. And if $\lambda_{2} \leq d \cdot(1-\varepsilon)$, then there is a set $S,|S| \leq|V| / 2$, such that the number of edges between $S$ and $\bar{S}$ is at most $d \cdot|S| \cdot \sqrt{2 \varepsilon}$. Similarly, Lemmas 2 and 3 characterizes the cases in which $\lambda_{n}$ is close to $-d$. If there is a set $S$ and a partition $(L, R)$ of $S$ such that $\leq \varepsilon d|S|$ edges incident on $S$ fail to be cut by the partition ${ }^{4}$, then, by sligthly generalizing the calculation in Lemma 2 we have $\lambda_{n} \leq d \cdot(-1+4 \varepsilon)$. And if $\lambda_{n} \geq d \cdot(-1+\varepsilon)$, then there is a set $S$ and a bipartition of $S$ into $L, R$ such that at most $d \cdot|S| \cdot \sqrt{2 \varepsilon}$ of the edges incident on $S$ fail to be cut by the bipartition.

If we define $h(G)$ to be the edge expansion of $G$,

$$
h(G)=\min _{S \subseteq V:|S| \leq|V| / 2} \frac{\operatorname{edges}(S, V-S)}{d|S|}
$$

then we have Cheeger's inequality

$$
\sqrt{2 \cdot \frac{d-\lambda_{2}}{d}} \geq h(g) \geq \frac{1}{2} \cdot \frac{d-\lambda_{2}}{d}
$$

Similarly, if we define the bipartiteness ratio number of a graph to be the smallest ratio between the number of failed edges and the quantity $d|S|$ :

$$
\beta(G):=\min _{S \subseteq V,(L, R) \text { partition of } \mathrm{S}} \frac{\operatorname{edges}(L)+\operatorname{edges}(R)+\operatorname{edges}(S, V-S)}{d|S|}
$$

then we have

$$
\sqrt{2 \cdot \frac{d-\left|\lambda_{n}\right|}{d}} \geq \beta(G) \geq \frac{1}{4} \cdot \frac{d-\left|\lambda_{n}\right|}{d}
$$

\footnotetext{
${ }^{4}$ Recall than an edge $(i, j)$ fails to cut the partition $L, R$ if both $i$ and $j$ are in $L$, or if both $i$ and $j$ are in $R$, or if $(i, j)$ connects $S$ with $\bar{S}$.
} 
There are examples in which both inequalities in (6) are tight.

If we take an odd cycle with $n$ vertices, then $\beta(G) \geq \frac{1}{n}$, because for every subset $S$ of vertices and for every bipartition of $S$ there is at least one failed edge, and the number of edges incident on $S$ is at most $n$. In an odd cycle, however, $d=2$ and $\left|\lambda_{n}\right|=2-O\left(1 / n^{2}\right)$, and so $\beta$ is as large as $\Omega\left(\sqrt{\left(d-\left|\lambda_{n}\right|\right) / d}\right)$.

To see the tightness of the other inequality, start from a $k$-regular expander such that, say, $\max \left\{\lambda_{2},\left|\lambda_{n}\right|\right\} \leq k / 2$. (Such graphs exist for constant $k$.) Then construct $G$ by taking the disjoint union of the edges of $G$ and the edges of a $k \cdot(1-\varepsilon) / \varepsilon$-regular bipartite graph, so that the resulting graph is $d$-regular with $d:=k / \varepsilon$. There is a cut that cuts all the edges of the bipartite graph, so $\beta(G) \leq \varepsilon$, but the smallest eigenvalue of $G$ is at least $-d+k / 2 \geq-d(1-\varepsilon / 2)$, meaning that $\beta$ is $O\left(\left(d-\left|\lambda_{n}(G)\right|\right) / d\right)$.

Our results, as stated in (6), are not just syntactically similar to Cheeger's inequality: There are also similarities between the proof of Cheeger's inequality and of Theorem 1. The analysis in Cheeger's inequality relies on the study of the quadratic form

$$
\sum_{i, j} A(i, j) \cdot\left(x_{i}-x_{j}\right)^{2}
$$

and it is based on the intuition that if $(7)$ is small compared to $\sum_{i} x_{i}^{2}$ then for most edges $(i, j)$ we have $x_{i} \approx x_{j}$.

Our analysis was based on the study of the quadratic form

$$
\sum_{i, j} A(i, j) \cdot\left(x_{i}+x_{j}\right)^{2}
$$

and the intuition that if (8) is small compared to $\sum_{i} x_{i}^{2}$ then for most edges we have $x_{i} \approx-x_{j}$.

\section{Relation to the Goemans-Williamson Relaxation}

The dual of the Goemans-Williamson relaxation is

$$
\begin{aligned}
& \min |E|-\frac{1}{4} \sum_{i} y_{i} \\
& \text { subject to } \\
& D+A-\operatorname{diag}\left(y_{1}, \ldots, y_{n}\right) \succeq 0
\end{aligned}
$$

We can see Lemma 2 as stating a special case of the weak duality fact that the cost of every feasible solution to (9) is an upper bound to the optimal cut in the graph.

Indeed, if the optimal cut is of size $>|E| \cdot(1-\varepsilon)$, then no solution of cost $\leq|E| \cdot(1-\varepsilon)$ can be feasible for (9). In particular, the solution $y_{i}=2 \varepsilon d_{i}$ has cost $1-\varepsilon$ and cannot be feasible, meaning that $D(1-2 \varepsilon)+A$ cannot be feasible, and there is a vector $x$ such that $x(D(1-2 \varepsilon)+A) x<0$.

In turn, Lemma 3 has the following primal dual interpretation: given a graph $G$ and a parameter $\varepsilon$, either algorithm 2 TSC finds $L, R$ such that at most a $4 \sqrt{\varepsilon}$ fraction of the edges incident on $L \cup R$ fail, or the solution $y_{i}:=2 \varepsilon d_{i}$ is feasible for (9). 
Given this premise, we can now view algorithm RECURSIVE-SPECTRAL-CuT as a primal-dual algorithm. (In the following analysis we assume for simplicity that $\delta=0$ in the algorithm of Theorem 1 , but the analysis is easily adjusted to account for $\delta$.)

Let, as before $\varepsilon_{0}=.015148 \cdots$ be the solution to $1 / 2\left(1-\varepsilon_{0}\right)=1-4 \sqrt{\varepsilon_{0}}$, consider the execution of RECURSive-Spectral-Cut on a given graph $G$, and divide (for the sake of analysis) the execution of the algorithm into two phases: Phase 1 is the sequence of steps in which the algorithm finds sets $L, R$ such that at most a $4 \sqrt{\varepsilon_{0}}$ fraction edges incident on $(L, R)$ fail; Phase 2 is the sequence of steps since the first time the above condition is violated until the end of the algorithm.

Let us write the adjacency matrix $A$ of $G$ as $A:=A^{(1)}+A^{(2)}$, where $A^{(2)}$ is the adjacency matrix of the residual graph at the start of Phase 2 , and $A^{(1)}$ is the matrix that accounts for all edges removed during Phase 1. Let also $D^{(1)}$ be the diagonal matrix of degrees of $A^{(1)}$ and similarly define $D^{(2)}$.

The graph with which we start Phase 2 is such that $D^{(2)} \cdot\left(1-2 \varepsilon_{0}\right)+A^{(2)} \succeq 0$. It is also certainly true that $D^{(1)}+A^{(1)} \succeq 0$, and so

$$
D+A-2 \varepsilon_{0} D^{(2)} \geq 0
$$

meaning that $y_{i}=2 \varepsilon_{0} d_{i}^{(2)}$ is feasible for (9).

If we denote by $m_{1}=\frac{1}{2} \sum_{i} d_{i}^{(1)}$ the number of edges in the graph $A^{(1)}$ and $m_{2}=\frac{1}{2} \sum_{i} d_{i}^{(2)}$ the number of edges in the graph $A^{(2)}$, then our algorithm cuts at least $\left(1-4 \sqrt{\varepsilon_{0}}\right) \cdot m_{1}+\frac{1}{2} \cdot m_{2}$ edges, and the semidefinite dual solution we have found proves that the optimum solution cuts at most $m_{1}+\left(1-\varepsilon_{0}\right) \cdot m_{2}$ edges, and notice that

$$
\left(1-4 \sqrt{\varepsilon_{0}}\right) \cdot m_{1}+\frac{1}{2} \cdot m_{2}=\frac{1}{2\left(1-\varepsilon_{0}\right)} \cdot\left(m_{1}+\left(1-\varepsilon_{0}\right) m_{2}\right)
$$

thus showing that the ratio of the cost of the solution found by the algorithm to the upper bound of the optimum implied by the dual solution is at least $1 /\left(2\left(1-\varepsilon_{0}\right)\right)=.507 \cdots$.

\section{Conclusions}

The motivating question for this work was to find a combinatorial interpretation of the quantity $d-\left|\lambda_{n}\right|$ in a $d$-regular graph, akin to the interpretation of $d-\lambda_{2}$ provided by the theory of edge expansion.

In establishing such an interpretation (in terms of the quantity that we call "bipartiteness ratio" in Section 5) we proved that a natural and easy-to-implement spectral algorithm performs nontrivially well with respect to the Max Cut problem. We expect the algorithm to perform well "in practice" and we are beginning an experimental study on which we will report in a follow-up paper.

It remains an interesting open question to find a "purely combinatorial" algorithm (namely, one not involving matrix computations) for Max Cut achieving an approximation factor better than $1 / 2$. 


\section{Acknowledgements}

I would like to thank an anonymous commenter for asking the question of the connection between spectral techniques and Max Cut, and Satyen Kale, James Lee and Salil Vadhan for providing helpful comments and references to the related literature.

\section{References}

[ACMM05] Amit Agarwal, Moses Charikar, Konstantin Makarychev, and Yury Makarychev. $O(\sqrt{\log n})$ approximation algorithms for min UnCut, min 2CNF deletion, and directed cut problems. In Proceedings of the 37th ACM Symposium on Theory of Computing, pages $573-581,2005.3$

[AK07] Sanjeev Arora and Satyen Kale. A combinatorial, primal-dual approach to semidefinite programs. In Proceedings of the 39th ACM Symposium on Theory of Computing, pages 227-236, 2007. 1, 15

[Alo86] Noga Alon. Eigenvalues and expanders. Combinatorica, 6(2):83-96, 1986. 2, 10

[ARV04] Sanjeev Arora, Satish Rao, and Umesh Vazirani. Expander flows and a $\sqrt{\log n}$ approximation to sparsest cut. In Proceedings of the 36th ACM Symposium on Theory of Computing, 2004. 2

[AS00] Noga Alon and Benny Sudakov. Bipartite subgraphs and the smallest eigenvalue. Combinatorics, Probability and Computing, 9:1-12, 2000. 3

[dlVKM07] Wenceslas Fernandez de la Vega and Claire Kenyon-Mathieu. Linear programming relaxations of maxcut. In Proceedings of the 18th ACM-SIAM Symposium on Discrete Algorithms, pages 53-61, 2007. 2

[FO05] Uriel Feige and Eran Ofek. Spectral techniques applied to sparse random graphs. Random Structures and Algorithms, 27(2):251-275, 2005. 3

[GW95] Michel X. Goemans and David P. Williamson. Improved approximation algorithms for maximum cut and satisfiability problems using semidefinite programming. Journal of the ACM, 42(6):1115-1145, 1995. Preliminary version in Proc. of STOC'94. 1

[Kho02] Subhash Khot. On the power of unique 2-prover 1-round games. In Proceedings of the 34th ACM Symposium on Theory of Computing, pages 767-775, 2002. 1

[KKMO04] Subhash Khot, Guy Kindler, Elchanan Mossel, and Ryan O'Donnell. Optimal inapproximability results for MAX-CUT and other two-variable CSPs? In Proceedings of the 45th IEEE Symposium on Foundations of Computer Science, pages 146-154, 2004. 1

[KW92] J. Kuczynski and H. Wozniakowski. Estimating the largest eigenvalues by the power and Lanczos algorithms with a random start. SIAM Journal on Matrix Analysis and Applications, 13:1094-1122, 1992. 5 
[MOO05] Elchanan Mossel, Ryan O'Donnell, and Krzysztof Oleszkiewicz. Noise stability of functions with low influences: invariance and optimality. In Proceedings of the 46th IEEE Symposium on Foundations of Computer Science, pages 21-30, 2005. 1

[OW08] R. O'Donnell and Y. Wu. An optimal SDP algorithm for Max-Cut, and equally optimal long code tests. In Proceedings of the 40th ACM Symposium on Theory of Computing, 2008. 1

[ST04] Daniel Spielman and Shang-Hua Teng. Nearly linear time algorithms for graph partitioning, graph sparsification, and solving linear systems. In Proceedings of the 36th ACM Symposium on Theory of Computing, pages 81-90, 2004. 3

[STT07] Grant Schoenebeck, Luca Trevisan, and Madhur Tulsiani. Tight integrality gaps for Lovasz-Schrijver lp relaxations of vertex cover and max cut. In Proceedings of the 39th ACM Symposium on Theory of Computing, pages 302-310, 2007. 2

[Tre01] Luca Trevisan. Non-approximability results for optimization problems on bounded degree instances. In Proceedings of the 33rd ACM Symposium on Theory of Computing, pages 453-461, 2001. 2, 15 


\section{A Appendix}

\section{A.1 Efficiency of the Arora-Kale Algorithm}

Arora and Kale [AK07] describe an algorithm for the Goemans-Williamson SDP relaxation of Max Cut which achieves an approximation ratio $1+o(1)$ and runs in time $\tilde{O}\left(D_{\max } \cdot|V|\right)$ given in input an unweighted multi-graph $G=(V, E)$ of maximum degree $D_{\max } \cdot{ }^{5}$ In particular, it is possible to find $(\alpha-o(1))$-approximate solutions to Max Cut in time $\tilde{O}\left(D_{\max } \cdot|V|\right)$, where $\alpha=.878 \cdots$ is the approximation ratio of the Goemans-Williamson algorithm.

In this section we show that, using the Arora-Kale algorithm and a reduction from [Tre01], it is possible to approximate Max Cut within $\alpha-o(1)$ in time $\tilde{O}(|V|+|E|)$ regardless of the degree distribution. ${ }^{6}$

Given the sparsification result discussed in Section 2, it is sufficient to prove the following theorem, which is implicit in [Tre01].

Theorem 6 There is a randomized algorithm $C$ and a deterministic algorithm $R$ with the following properties.

Given a graph $G=(V, E)$, algorithm $C$ constructs in $\tilde{O}(|V|+|E|)$ time a graph $G^{\prime}=\left(V^{\prime}, E^{\prime}\right)$ of maximum degree $\tilde{O}(1)$ with $\left|V^{\prime}\right|=2|E|$ vertices, such that the following happens with high probability: (i) $\operatorname{maxcut}\left(G^{\prime}\right) \geq \operatorname{maxcut}(G)-o(1)$, and (ii) given an arbitrary solution $S^{\prime} \subseteq V^{\prime}$ of cost $c$ in $G^{\prime}$, algorithm $R$ constructs in $\tilde{O}(|V|+|E|)$ time a solution $S \subseteq V$ of cost $\geq c-o(1)$ for $G$.

Proof: We sketch how the argument in [Tre01] applies to Max Cut.

Define the weighted graph $\hat{G}=(\hat{V}, \hat{E})$ as follows. (This graph will only be used in the analysis, and not explicitely constructed in the reduction.) For every vertex $v \in V$ of degree $d_{v}, \hat{V}$ contains $d_{v}$ copies of $v$; for every edge $(u, v)$ in $E$, we have $d_{u} \cdot d_{v}$ edges $(\hat{u}, \hat{v})$ in $E^{\prime}$, one for every copy $\hat{u}$ of $u$ and for every copy $\hat{v}$ of $v$, each such edge having weight $1 /\left(d_{u} \cdot d_{v}\right)$.

We claim that approximating Max Cut in $G$ is equivalent to approximating Max Cut in $\hat{G}$. First, it should be clear that if $(S, V-S)$ is a cut in $G$ of cost $c$, then if we define $\hat{S} \subseteq \hat{V}$ to be the set of all copies of vertices in $S$, then $(\hat{S}, \hat{V}-\hat{S})$ is a cut of cost $c$ in $\hat{G}$. On the other hand, if $(\hat{S}, \hat{V}-\hat{S})$ is a cut of cost $c$, then consider the distribution over cuts in $G$ in which a vertex $v$ is picked to be in $S$ with probability proportional to the fraction of copies of $v$ which are in $\hat{S}$; the expected fraction of cut edges in $G$ is exactly $c$, and using the method of conditional expectations we can find a cut of cost at least $c$ in linear time.

The graph $G^{\prime}$ is obtained by sampling with replacement $\tilde{O}(|\hat{V}|)=\tilde{O}(|V|+|E|)$ edges from $\hat{E}$, using the distribution in which an edge is sampled with probability proportional to its weight. As discussed in Section 2, it follows from Chernoff bounds that a solution of cost $c$ in $G^{\prime}$ has cost $c \pm o(1)$ in $\hat{G}$.

It remains to discuss the complexity of sampling $G^{\prime}$ : to sample one edge, we first pick a random edge $(u, v)$ of $G$, and then we pick at random one of the copies $\hat{u}$ of $u$ and one of the copies $\hat{v}$

\footnotetext{
${ }^{5}$ The Arora-Kale result is more general, but this statement is sufficient for our purpose

${ }^{6}$ The running time can be reduced to $\tilde{O}(|V|)$ if the representation of the graph is such that a random edge can be sampled in $\tilde{O}(1)$ time, and the degree of a given vertex can be found in $\tilde{O}(1)$ time.
} 
of $v$; this distribution is equivalent to randomly sampling one of the edges of $\hat{G}$ with probabiltiy proportional to its weight. After $O(|V|+|E|)$ time preprocessing, each edge of $G^{\prime}$ can be sampled in constant time. ${ }^{7}$

\footnotetext{
${ }^{7}$ The point of this discussion is that $\hat{G}$ may have $\Omega\left(|V|^{2}\right)$ edges even if $|E|=O(|V|)$, for example if there are two vertices of degree $|V|-1$. This means that it is not possible to explicitly construct $\hat{G}$ in $\tilde{O}(|V|+|E|)$ time, and so one must sample edges from $\hat{G}$ without explicitly constructing $\hat{G}$.
} 